\section{(2) OPEN ACCESS}

\title{
Use of, and likelihood of using, HIV pre-exposure prophylaxis among men who have sex with men in Europe and Central Asia: findings from a 2017 large geosocial networking application survey
}

\author{
Adam Bourne, ${ }_{1}^{1}$ Beatrice Alba, ${ }_{1}$ Alex Garner, ${ }^{2}$ Gianfranco Spiteri, ${ }^{3}$ Anastasia Pharris, ${ }^{3}$ \\ Teymur Noori ${ }^{3}$
}

${ }^{1}$ Australian Research Centre in Sex, Health \& Society, La Trobe University, Melbourne, Victoria, Australia

${ }^{2}$ Hornet Gay Social Network San Francisco, California, USA ${ }^{3}$ European Centre for Disease Prevention and Control, Solna Sweden

\section{Correspondence to} Adam Bourne, Australian Research Centre in Sex, Health \& Society, La Trobe University, Melbourne, Victoria 3086, Australia; a.bourne@latrobe. edu.au

Received 31 May 2018 Revised 2 October 2018 Accepted 10 October 2018 Published Online First 5 January 2019

\section{Check for updates}

(c) Author(s) (or their employer(s)) 2019. Re-use permitted under CC BY-NC. No commercial re-use. See rights and permissions. Published by BMJ.

To cite: Bourne $A$, Alba B, Garner A, et al. Sex Transm Infect 2019:95:187-192.

\begin{abstract}
Objective Currently, seven European countries provide HIV pre-exposure prophylaxis (PrEP) through public health services, although there are numerous reports of off-licence use. The objective of this study was to examine current use of PrEP, likelihood of future use and indicators of potential PrEP candidacy among an opportunistic sample of men who have sex with men in Europe and Central Asia.
\end{abstract}

Methods A survey was sent out in eight languages to users of the Hornet gay networking application in July and August 2017. Descriptive statistics present proportions of PrEP use, while factors associated with PrEP use are examined using logistic and linear regressions.

Results of 12053 participants whose responses came from 55 European and Central Asian countries, 10764 $(89 \%)$ were not living with diagnosed HIV. Among these HIV-negative/untested men, 10.1\% ( $n=1071)$ were currently taking PrEP or had done so within the previous 3 months. Current or recent PrEP users were significantly more likely to have taken postexposure prophylaxis (adjusted OR (AOR) $=16.22(95 \% \mathrm{Cl} 13.53$ to 19.45$))$ or received an STI diagnosis (AOR=4.53 $(95 \% \mathrm{Cl} 3.77$ to 5.44)) in the previous 12 months than those who had not. Most commonly, these men obtained PrEP from a physician (28.1\%) or the internet (24.8\%), while 33.6\% had not disclosed PrEP use to their doctor. Men reporting happiness with their sex life were more likely to have taken $\operatorname{PrEP}(A O R=1.73(95 \% \mathrm{Cl} 1.59$ to 1.89)). Nearly a quarter $(21.5 \%)$ of those not on PrEP said they were likely to use it in the next 6 months.

Conclusions The majority of men using, or intending to use, PrEP appear to have a risk profile consistent with emerging guidance. $A$ large proportion of these men are accessing PrEP outside of traditional healthcare settings, posing a challenge for routine monitoring.

\section{BACKGROUND}

Pre-exposure prophylaxis (PrEP) has the potential to radically alter the trajectory of the HIV epidemic. In Europe, sex between men remains the primary mode of HIV transmission ${ }^{1}$ with $40 \%$ of new HIV diagnoses among this population in 2016. While the use of condoms among gay, bisexual and other men who have sex with men (GBMSM) has mitigated HIV transmission, ${ }^{2}$ they have proved insufficient in stemming a tide of new infections. Numerous clinical trials have now documented that PrEP can reduce the likelihood of acquiring HIV among negative men by at least $86 \%,{ }^{34}$ and likely closer to $99 \%$ when adherence to the medication is high. In May 2015, the European Centre for Disease Prevention and Control (ECDC) recommended that European Union/European Economic Area Member States should give consideration to integrating PrEP into their existing HIV prevention package for those most at risk of HIV infection, starting with GBMSM. ${ }^{5}$ The WHO now recommends PrEP be provided to GBMSM, recognising their status as a most-at-risk population. ${ }^{6}$ In July 2016, the European Medicines Agency gave a positive opinion on the use of PrEP (emtricitabine/ tenofovir disoproxil) for prevention to reduce the transmission of $\mathrm{HIV}^{7}$

Despite the convincing evidence base for PrEP efficacy, roll-out and national provision to those most at risk of HIV remain relatively low across Europe and Central Asia, in part driven by costs of the medication and the required wraparound clinical care. ${ }^{8}$ PrEP is currently available via national healthcare systems in France, Norway, Belgium, Luxembourg, Portugal and Scotland. In other countries, for example, Germany, Austria, Poland and Armenia, a course of PrEP is available for between $€ 15$ and $€ 50$ per month. PrEP is available in the Netherlands with a prescription from a doctor but is not currently covered by health insurers; hence, the cost is borne by the patient (currently $€ 54$ for a 4-week course), unless they are part of an ongoing demonstration project. In Ireland, a generic form of PrEP has been available in pharmacies with a doctor's prescription since early December 2017. In England, Wales, Ukraine and Azerbaijan, PrEP is available to a limited number of men via demonstration projects, while in all other countries of Europe and Central Asia PrEP is not currently available by any national health service or clinical trial mechanism. ${ }^{9}$

However, significant advocacy efforts from community initiatives such as PrEPster, I want PrEP Now and QuieroPrEPYa have contributed to some 
men accessing PrEP outside of formal prescription mechanisms. A number of websites and advocacy platforms across the continent have sought to facilitate access to PrEP online, typically sourced from pharmacies in Thailand and South Africa. I want PrEP Now, for example, provides information on where PrEP can be obtained online, to which countries it can be delivered and, crucially, information on therapeutic drug monitoring. In partnership with central London sexual health clinic, 56 Dean Street, they have sought to identify manufacturers and batches of generic PrEP that demonstrate adequate levels of tenofovir or emtricitabine (the active ingredients of PrEP) in their blood. ${ }^{10}$

While demonstration projects provide some indication of the number of men using PrEP in those countries where these are in operation, there are few other national or regional estimates of the proportion of GBMSM using PrEP that take into account those accessing it informally in online environments. Crucially, there are also few national or regional assessments of the willingness to use PrEP, data which are fundamental to the resourcing, design and delivery of the medication to those at highest risk of acquiring HIV. This study sought to examine the current use of PrEP, likelihood of future use and indicators of potential PrEP candidacy in an opportunistic sample of European and Central Asian GBMSM.

\section{METHODS \\ Design}

Between 17 June and 16 August 2017, Hornet Gay Social Network, in collaboration with the ECDC, sent out an anonymous self-completed questionnaire in eight languages (Arabic, English, French, German, Italian, Portuguese, Russian and Spanish) to users of the Hornet application. Hornet is a free gay social network that connects around 25 million GBMSM, including large numbers of men in Europe and Central Asia. Participants were eligible if they self-reported to be male, were aged 18 and above, identified as a man who has sex with other men, and lived within the 55 countries of Europe and Central Asia according to the United Nations country classification system. Participants who did not report sex with other men or self-identify as men who had sex with men were excluded from the survey. The survey was programmed and delivered via SurveyMonkey and served as a direct in-app message based on the language of the user's Hornet account (eg, users who accessed the Arabic version of Hornet were sent the survey link in Arabic). Clicking on the message took participants to our survey landing page, which provided full details of the survey, highlighting they were under no obligation to take part and were asked to indicate their consent. No financial incentives were offered.

\section{Measures}

The survey was deliberately designed to be brief to facilitate completion, and thus collected only basic demographic information, including country of residence and age and diagnosed HIV status. To ensure comprehension, all men were told that PrEP (Pre-Exposure Prophylaxis) is a proven effective method to prevent HIV. It needs to be taken before having sex and, at present, is most commonly taken on a daily basis. They were asked to indicate whether they were currently using PrEP or whether they had done so within the previous 3 months. Those who indicated such current or recent use were asked where they obtained it (ticking all those options that applied) and whether their sexual health physician or doctor knew they were taking PrEP. All were then asked To what extent do you agree with the following statement: 'I am very likely to use PrEP in the next 6 months', with a 5-point response scale from 1 (strongly disagree) to 5 (strongly agree), to measure their likelihood of future PrEP use.

Additional questions sought to establish the behavioural sexual risk profile of respondents by establishing use of postexposure prophylaxis (PEP) within the previous 12 months, diagnosis with an STI within the previous 12 months (yes/no/not sure), and use of mephedrone, gamma hydroxybutyrate/gamma butyrolactone, ketamine or crystal methamphetamine during sex with men (otherwise known as 'chemsex') within the previous 3 months. PEP use, recent STI diagnoses and engagement in chemsex are all indicators of HIV risk behaviour and commonly associated with an HIV diagnosis. ${ }^{11} 12$ Recognising the significant improvement in sexual well-being and reduction in anxieties during sex when using PrEP documented by previous qualitative research, ${ }^{13}$ we sought to assess and correlate sexual happiness by a 5 -point Likert scale with permissible responses from very unhappy to very happy.

\section{Analysis}

Men who reported they have HIV were excluded for the purposes of these analyses. Given the evidence and awareness of using PrEP intermittently, men who indicated they were currently using PrEP or had done so in the previous 3 months were merged into one group for analysis. Current PrEP use was analysed using binary logistic regression, and the likelihood of PrEP use in the next 6 months was analysed using linear regression. Predictor variables were PEP use in the past 12 months, STI diagnosis in the past 12 months, engaging in chemsex in the last 3 months and happiness with sex life. Each analysis was also conducted while adjusting for age and country of residence, and the analysis of the likelihood of PrEP use in the next 6 months was also adjusted for current PrEP use. All analyses were conducted using SPSS V.24.

\section{RESULTS}

\section{Participant characteristics}

Following their online informed consent, 12053 men from Europe or Central Asia completed the survey (974 participants were excluded because they were not from Europe or Central Asia), of whom 1289 reported they were HIV-positive, a prevalence rate of $10.7 \%$. The 10764 HIV-negative or untested men who completed the survey came from 54 countries of Europe and Central Asia. Of this sample, 202 men did not indicate whether or not they used PrEP, and were therefore excluded from further analysis. This left a final sample of 10562 participants. The highest proportions of the sample included men from France $(n=2544,24.1 \%)$, Russia $(n=2393,22.7 \%)$, UK $(n=1414$, $13.4 \%)$, Italy $(n=797,7.5 \%)$ and Turkey $(n=617,5.8 \%)$. More than a quarter of participants $(n=3065,29.0 \%)$ were aged 18-25 and nearly half $(\mathrm{n}=5043,47.8 \%)$ were aged between 26 and 40. A full breakdown of the sample by age and country of residence is shown in table 1 . In terms of HIV risk indicators, 7.9\% $(n=773)$ had used PEP in the previous 12 months, $19.4 \%$ $(n=1261)$ had received an STI diagnosis in the past 12 months and $6.8 \%(n=622)$ had engaged in chemsex within the previous 3 months. In total, $55.5 \%$ were happy or very happy with their sex life (table 2).

\section{Current or recent use of PreP}

One in 10 men $(n=1071,10.1 \%)$ were currently taking PrEP or had done so in the previous 3 months. This differed significantly according to age, $\chi^{2}(6)=-51.47, \mathrm{p}<0.001$, and country 
Table 1 Number of participants using pre-exposure prophylaxis (PreP) (or used in the previous 3 months) and the likelihood of future PreP use by age and country of residence

\begin{tabular}{|c|c|c|c|c|}
\hline & n (\%) & Currently taking PrEP, n (\%) & Not currently taking PrEP, n (\%) & Likelihood of future PrEP use, M (SD) \\
\hline \multicolumn{5}{|l|}{ Age } \\
\hline $18-25$ & $3065(29.0)$ & $219(7.1)$ & $2846(92.9)$ & $2.89(1.12)$ \\
\hline $26-30$ & 2095 (19.9) & $219(10.5)$ & $1876(89.5)$ & $2.91(1.19)$ \\
\hline $31-40$ & 2948 (27.9) & $324(11.0)$ & $2624(89.0)$ & $2.91(1.22)$ \\
\hline $41-50$ & $1680(15.9)$ & $222(13.2)$ & $1458(86.8)$ & $3.02(1.30)$ \\
\hline $51-60$ & $620(5.9)$ & $65(10.5)$ & $555(89.5)$ & $2.98(1.26)$ \\
\hline $61-70$ & $123(1.2)$ & $16(13.0)$ & $107(87.0)$ & $2.86(1.31)$ \\
\hline$>70$ & $20(0.2)$ & $2(10.0)$ & $18(90.0)$ & $3.12(1.05)$ \\
\hline Total & $n=10551$ & $1067(10.1)$ & $9484(89.9)$ & $2.93(1.21)$ \\
\hline \multicolumn{5}{|l|}{ Country of residence } \\
\hline France & $2544(24.1)$ & $281(11.0)$ & $2263(89.0)$ & $3.08(1.29)$ \\
\hline Russia & $2393(22.7)$ & $195(8.1)$ & 2198 (91.9) & $2.84(1.06)$ \\
\hline UK & $1414(13.4)$ & $146(10.3)$ & $1268(89.7)$ & $2.93(1.30)$ \\
\hline Italy & $797(7.5)$ & $50(6.3)$ & $747(93.7)$ & $2.60(1.11)$ \\
\hline Turkey & $617(5.8)$ & $99(16.0)$ & $518(84.0)$ & $3.13(1.13)$ \\
\hline Ukraine & $374(3.5)$ & $62(16.6)$ & $312(83.4)$ & $3.01(1.10)$ \\
\hline Germany & $309(2.9)$ & $30(9.7)$ & $279(90.3)$ & $2.75(1.33)$ \\
\hline Belgium & $209(2.0)$ & $19(9.1)$ & $190(90.9)$ & $3.29(1.13)$ \\
\hline Belarus & $195(1.8)$ & $17(8.7)$ & $178(91.3)$ & $2.92(1.03)$ \\
\hline Spain & $183(1.7)$ & $13(7.1)$ & $170(92.9)$ & $2.76(1.15)$ \\
\hline The Netherlands & $158(1.5)$ & $21(13.3)$ & $137(86.7)$ & $3.01(1.29)$ \\
\hline Denmark & $145(1.4)$ & $14(9.7)$ & $131(90.3)$ & $2.65(1.21)$ \\
\hline Switzerland & $134(1.3)$ & $8(6.0)$ & $126(94.0)$ & $2.65(1.34)$ \\
\hline Portugal & $131(1.2)$ & $6(4.5)$ & $125(95.4)$ & $3.07(1.10)$ \\
\hline Sweden & $117(1.1)$ & $18(15.4)$ & 99 (84.6) & $3.05(1.36)$ \\
\hline Other & $842(8.0)$ & $92(10.9)$ & $750(89.1)$ & $2.83(1.16)$ \\
\hline Total & $n=10562$ & $\mathrm{n}=1071(10.1)$ & $\mathrm{n}=9491(89.9)$ & $2.93(1.21)$ \\
\hline
\end{tabular}

of residence, $\chi^{2}(15)=-81.92, \mathrm{p}<0.001$. Men aged $41-50$ were most likely to use PrEP (13.2\%), whereas men aged 18-25 were least likely (7.1\%). Use of PrEP was highest among men from Ukraine (16.6\%), Turkey (16.0\%), Sweden (15.4\%), the Netherlands (13.3\%), France (11.0\%) and the UK (10.3\%). When controlling for age and country of residence as covariates, the adjusted OR (AOR) of current or recent PrEP use was found to be significantly higher for men who had used PEP in the previous 12 months $(\mathrm{AOR}=16.22$ (95\% CI 13.53 to 19.45$)$ ), men who had received an STI diagnosis within the previous 12 months $(\mathrm{AOR}=4.53$ (95\% CI 3.77 to 5.44)), men who had engaged in chemsex during the last 3 months (AOR=7.19 (95\% CI 5.86 to

Table 2 Binary logistic regressions of predictors of current PreP use (or use in the previous 3 months)

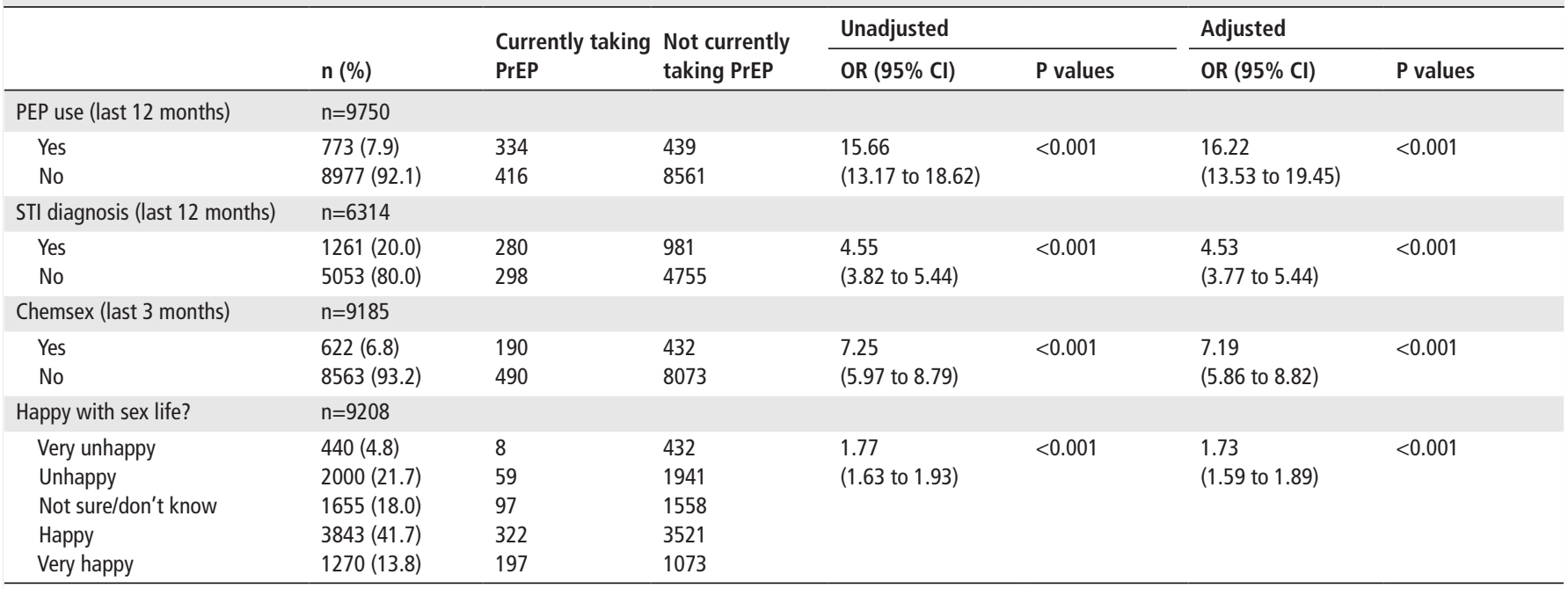

Notes: Adjusted analyses include age and country of residence (reference group=France) as covariates. Participants who responded with 'unsure' to having an STI diagnosis were excluded from this analysis.

PEP, post-exposure prophylaxis; PrEP, pre-exposure prophylaxis. 
Table 3 Obtaining PreP and disclosure of PreP use to medical professionals

\begin{tabular}{|c|c|c|c|c|c|c|c|c|c|}
\hline \multirow{2}{*}{$\begin{array}{l}\text { Country of } \\
\text { residence }\end{array}$} & \multirow[b]{2}{*}{ On PrEP } & \multicolumn{6}{|c|}{ Where was PrEP obtained? n (\%) } & \multicolumn{2}{|c|}{$\begin{array}{l}\text { Is your sexual health physician } \\
\text { doctor aware? } \mathrm{n}(\%)\end{array}$} \\
\hline & & Physician & Research study & Internet & Friend & Taken as PEP & Other & Yes & No \\
\hline France* & 281 & $175(62.3)$ & $32(11.4)$ & $15(5.3)$ & $21(7.5)$ & $20(7.1)$ & $24(8.5)$ & $170(67.5)$ & $82(32.5)$ \\
\hline Russia & 195 & $30(15.4)$ & $8(4.1)$ & $30(15.4)$ & $23(11.8)$ & $4(2.1)$ & $4(2.1)$ & $38(49.4)$ & 39 (50.6) \\
\hline $\mathrm{UK}^{*} \dagger$ & 146 & $8(5.5)$ & $18(12.3)$ & $112(76.7)$ & $6(4.1)$ & $3(2.1)$ & $7(4.8)$ & $110(78.0)$ & $31(22.0)$ \\
\hline Italy & 50 & $7(14.0)$ & $9(18.0)$ & $9(18.0)$ & $2(4.0)$ & $2(4.0)$ & $1(2.0)$ & $18(69.2)$ & $8(30.8)$ \\
\hline Turkey & 99 & $13(13.1)$ & $6(6.1)$ & 16 (16.2) & $14(14.1)$ & $7(7.1)$ & $5(5.1)$ & $28(58.3)$ & $20(41.7)$ \\
\hline Ukrainet & 62 & $10(16.1)$ & $4(6.5)$ & $11(17.7)$ & $8(12.9)$ & $2(3.2)$ & $0(0)$ & $14(66.7)$ & $7(33.3)$ \\
\hline Germany & 30 & $5(16.7)$ & $2(6.7)$ & $13(43.3)$ & $4(13.3)$ & $1(3.3)$ & $3(10.0)$ & $13(65.0)$ & $7(35.0)$ \\
\hline Belgium* & 19 & $9(47.4)$ & $4(21.1)$ & $1(5.3)$ & $1(5.3)$ & $0(0)$ & $3(15.8)$ & $13(86.7)$ & $2(13.3)$ \\
\hline Belarus & 17 & $3(17.6)$ & $1(5.9)$ & $4(23.5)$ & $3(17.6)$ & $1(5.9)$ & $1(5.9)$ & $5(50.0)$ & $5(50.0)$ \\
\hline Spain & 13 & $1(7.7)$ & $2(15.4)$ & $2(15.4)$ & $2(15.4)$ & $0(0)$ & $1(7.7)$ & $5(83.3)$ & $1(16.7)$ \\
\hline $\begin{array}{l}\text { The } \\
\text { Netherlands } \neq\end{array}$ & 21 & $4(19.0)$ & $7(33.3)$ & $5(23.8)$ & $1(4.8)$ & $0(0)$ & $2(9.5)$ & $13(72.2)$ & $5(27.8)$ \\
\hline Denmark & 14 & $3(21.4)$ & $5(35.7)$ & $1(7.1)$ & $3(21.4)$ & $0(0)$ & $1(7.1)$ & $12(100)$ & $0(0)$ \\
\hline Switzerland & 8 & $5(62.5)$ & $0(0)$ & $5(62.5)$ & $0(0)$ & $0(0)$ & $0(0)$ & $4(50.0)$ & $4(50.0)$ \\
\hline Portugal* & 6 & $1(16.7)$ & $1(16.7)$ & $2(33.3)$ & $2(33.3)$ & $1(16.7)$ & $0(0)$ & $4(100)$ & $0(0)$ \\
\hline Sweden & 18 & $2(11.1)$ & $0(0)$ & $13(72.2)$ & $1(5.6)$ & $0(0)$ & $1(5.6)$ & $5(29.4)$ & $12(70.6)$ \\
\hline Other & 92 & $25(27.2)$ & $9(9.8)$ & $27(29.3)$ & $17(18.5)$ & $3(3.3)$ & $7(7.6)$ & $34(59.6)$ & $23(40.4)$ \\
\hline Total & 1071 & $301(28.1)$ & $108(10.1)$ & $266(24.8)$ & $108(10.1)$ & $44(4.1)$ & $60(5.6)$ & $486(66.4)$ & $246(33.6)$ \\
\hline
\end{tabular}

Note in the case of UK, PrEP is available in the national health system in Scotland, only via demonstrations or clinical trials in England and Wales, and not available in Northern Ireland.

*PrEP available as part of the national health system at the time of survey.

TPrEP available to a limited extent through demonstration projects or clinical trials.

$\ddagger$ PrEP available at state-subsidised cost at the time of survey.

PrEP, pre-exposure prophylaxis.

$8.82)$ ) and men who were happier with their sex life (AOR $=1.73$ (95\% CI 1.59 to 1.89$)$ ) (table 2 ).

\section{Obtaining PreP and disclosure of PreP use to medical professionals}

Men were most likely to be obtaining PrEP from a physician $(28.1 \%)$ or the internet $(24.8 \%)$ (table 3$)$. In countries with samples of 30 or more men on PrEP, internet acquisition of PrEP was reported to be highest among those from the UK (76.7\%) and Germany (43.3\%). Comparatively, internet acquisition of PrEP was low among men from France (5.3\%), while physician-acquired PrEP was highest among men from this country $(62.3 \%)$. Overall, approximately one-third of PrEP users (33.6\%) had not disclosed their PrEP use to their doctor.

\section{Likelihood of future PreP use}

Overall, 2547 (33.2\%) men agreed or strongly agreed that they were likely to use PrEP in the next 6 months. Of the men who were not current PrEP users ( $\mathrm{n}=9491), 1926$ (21.5\%) agreed or strongly agreed that they were likely to use PrEP in the next 6 months. Of the 731 men who were currently using PrEP and who answered this question, $621(85.0 \%)$ agreed or strongly agreed that they were likely to use PrEP in the next 6 months. Scores on the likelihood of using PrEP in the next 6 months range from 1 (strongly disagree) to 5 (strongly agree), with a mean score of $2.93(\mathrm{SD}=1.21)$ among the entire sample and $2.80(\mathrm{SD}=1.14)$ among those who were not currently using PrEP. Scores on the likelihood of future PrEP use differed according to age, $F(6,9665)=2.64, \mathrm{p}=0.02$, and country of residence, $F(15$, $9664)=11.55, \mathrm{p}<0.001$. When adjusting for age, country of residence and current PrEP use as covariates, the likelihood of future PrEP use was significantly higher for men who had used PEP in the previous 12 months ( $b=1.01$ (95\% CI 0.92 to 1.09$)$ ), men who had received an STI diagnosis within the previous 12 months ( $b=0.42$ (95\% CI 0.35 to 0.50$)$ ), men who had engaged in chemsex during the last 3 months ( $b=0.78$ (95\% CI 0.68 to $0.88)$ ) and men who were happier with their sex life $(b=0.09$ (95\% CI 0.06 to 0.11$)$ ). The results for these linear regressions are shown in table 4.

\section{DISCUSSION}

This study identified that 1 in 10 men in a very large online survey were currently using PrEP and that a significant proportion were accessing the medication outside of formal health systems. Those currently using PrEP commonly reported other behaviours that put them at risk of acquiring HIV. Among those not currently using PrEP, a high willingness to do in the future was reported across all countries.

This proportion of current PrEP use came despite the fact that PrEP was not available via national health systems in the vast majority of these countries. More than one-third of those currently using PrEP, or who had done so recently, accessed the medication outside of formal clinical prescribing mechanisms, such as from a physician or within the confines of a PrEP-related research study. Nearly a quarter of men in total, and a considerably higher proportion of men in some countries, were using PrEP that had been acquired online, while a further $10 \%$ were acquiring it from friends. Furthermore, even as efforts continue across the continent to devise or revise PrEP clinical prescribing guidance, it is important to recognise that one-third of GBMSM within this sample have reportedly accessed and used the medication without informing their sexual health physician or other doctor. This does not necessarily mean that such men were not engaging in regular HIV or STI testing (they may have done so without disclosing their PrEP use), but does pose more of a challenge for the routine monitoring of kidney function. Further research that examines the 
Table 4 Linear regressions of predictors of likelihood of using PreP in the next 6 months

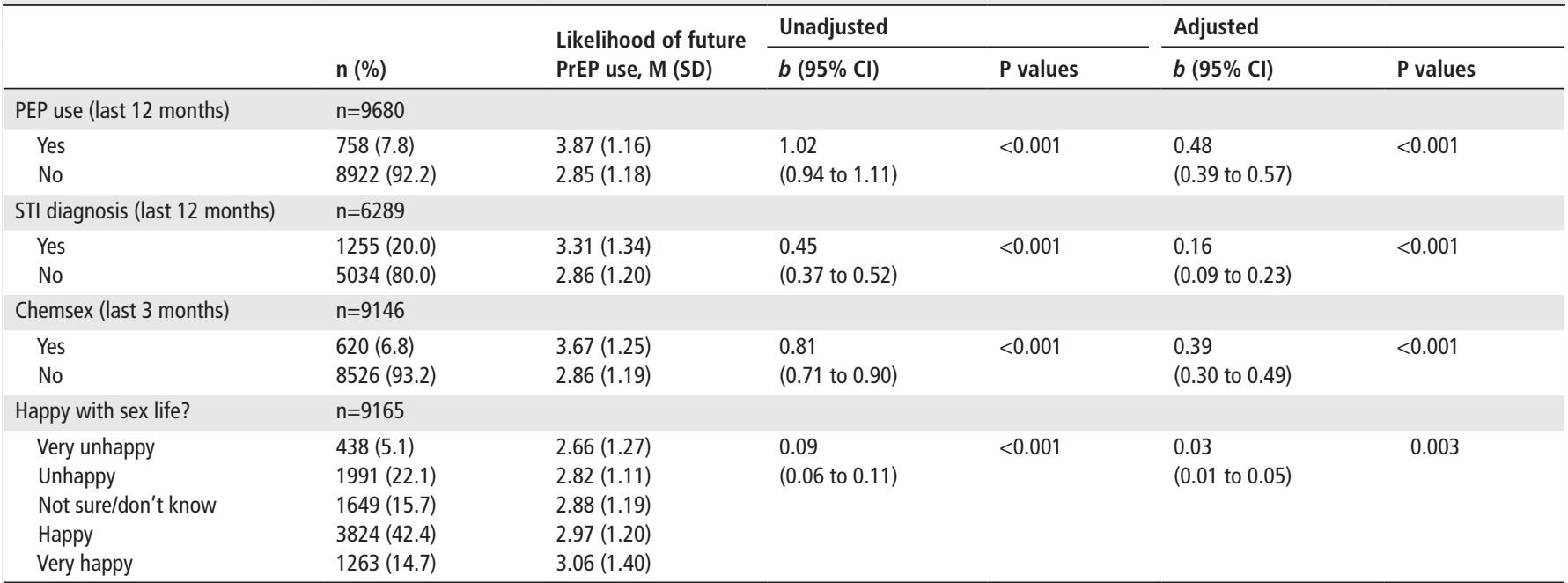

Note: Adjusted analyses include age, country of residence (reference group=France) and current PrEP use as covariates. Participants who responded with 'unsure' to having an STI diagnosis were excluded from this analysis.

PEP, post-exposure prophylaxis; PrEP, pre-exposure prophylaxis.

HIV/STI testing practices and other health monitoring of people accessing PrEP online is warranted.

Several organisations across the continent have sought to ensure the safety and efficacy of online-acquired PrEP by batch testing and by monitoring in partnership with sexual health clinics. ${ }^{10}$ Most clinical guidance relating to PrEP recommends testing for HIV and other STIs every 3 months, both to ensure efficacy of the medication in preventing seroconversion, but also to prevent the spread of STIs in a context where anal intercourse with condoms may be less likely. In a PrEP demonstration project in the USA, overall STI incidence was 90 per 100 person-years, providing a clear rationale for regular screening among PrEP users. ${ }^{14}$ With imprecise data on the date of PrEP initiation and time of STI testing and diagnosis, it is not possible from this survey to decipher whether men were testing for STIs while already on PrEP. Regardless, the lack of disclosure of PrEP use to sexual health physicians or other doctors means that conversations about the necessity of regular screening are less likely to occur. A lack of disclosure also poses a challenge for regular serum creatinine checks for kidney function, a common feature of PrEP prescribing guidelines. ${ }^{3}$

There are currently no other published European-wide estimates of PrEP use against which we can compare; however, data from the USA indicate that 136000 men who have sex with men (MSM) were taking a branded version of tenofovir disoproxil fumarate/ emtricitabine for HIV prevention in the first quarter of $2017 .^{15}$ This reflects a very significant increase in PrEP uptake in the USA between 2012 and 2016. The proportion of people using PrEP outside of formal clinical prescribing reported in the current study is significantly higher than observed in a 2016 community survey of 15880 people (including both men and women) from across 12 European countries, where only 303 (1.9\%) reported informal use of PrEP. ${ }^{16}$ However, the result of the 2016 study is perhaps skewed by a large proportion of the sample residing in Germany, where there is a more restrictive legal environment for the online purchase and import of PrEP. A recently published meta-analysis of all published studies on PrEP uptake found that by $2017,10.7 \%$ of participants reported ever using PrEP. ${ }^{17}$ The odds of reporting such use approximately doubled each year, highlighting the importance of routine PrEP monitoring in a period of rapid expansion. An analysis of data from an online survey of MSM in Ireland, ${ }^{18}$ which applied French PrEP initiation criteria, ${ }^{19}$ suggests that an estimated
$23 \%$ of MSM in the country would be eligible for PrEP. If applied to other countries in Europe, it would appear there is significant scope for an enhanced uptake and use of PrEP among MSM in the region.

Regardless of the means by which men in this sample were acquiring PrEP, the findings of the current study indicate that those men currently using PrEP would be deemed 'high or medium risk' for acquiring HIV according to national and international PrEP prescribing guidelines. ${ }^{620}$ The odds of engaging in chemsex, having received an STI diagnosis or previous use of PEP were all significantly higher among men using PrEP compared with those who were not. In this regard, we believe that the men using PrEP in this study represent ideal PrEP candidates, perhaps indicating an accurate self-perception of their risk of acquiring HIV. A similar correlation is also observed in relation to perceived likelihood of using PrEP in the near future: men with experience of these higher risk behaviours were significantly more likely to indicate willingness to use PrEP in the future. The extent to which this risk perception has been influenced by community-level PrEP initiatives or other health promotion programmes could be the focus of future study.

Within this sample, men who currently use PrEP were more likely to be happy with their sex life than men who were not. We make no claims as to a causal relationship, but this finding does align with prior qualitative research ${ }^{13}$ that indicates that GBMSM using PrEP experience less anxiety in their context of their sex lives. Many GBMSM have lived with a concern or fear of HIV throughout their lives, ${ }^{21} 22$ and PrEP provides the means to calm such anxieties. The implications of this should not be understated. Not only might PrEP enable broader sexual well-being for GBMSM, but such findings can also be valuable in the framing of PrEP health promotion interventions and PrEP demand creation activities among men who may benefit from PrEP but who remain uncertain about its value for them. ${ }^{23} 24$

This survey was delivered via one large geosocial networking application popular among GBMSM. While accessible in eight languages, there are numerous other languages of Europe and Central Asia that were not represented, which may have posed a challenge to completion for some. In an attempt to enable swift completion, only basic demographic information was collected, meaning that more complex analyses controlling for potential mediators (such as income or beliefs in PrEP efficacy) could not 
be performed. In addition, an overall HIV prevalence rate in the sample of $10.7 \%$ may reflect a higher sexual risk profile of men using Hornet, or of gay geosocial networking apps more generally; however, this would also indicate an ideal target group for PrEP-related interventions. The provision and uptake of PrEP is developing across the continent and more countries have provided access to PrEP now than was the case when the survey was conducted, reinforcing a need for regular monitoring of this dynamic environment. It is also important to note that willingness to use PrEP was only examined using a single-item measure, whereas other multi-item scales have shown reliability in assessing PrEP acceptability. ${ }^{25}$ These limitations notwithstanding, these data provide a unique and valuable snapshot of PrEP uptake and use at a critical time in the national and regional roll-out of this new HIV prevention technology.

\section{Key messages}

- Approximately 1 in 10 men were using pre-exposure prophylaxis (PrEP), with significantly higher proportions or users in France, Russia and the UK.

- Nearly a quarter of those using PrEP had acquired PrEP online, while a further $10 \%$ had acquired it from friends.

- Regardless of the means by which they were accessing PrEP, analysis of their HIV risk profile indicates they are ideal candidates for doing so.

- Men currently using PrEP were significantly more likely to report happiness with their sex life than men who were not.

\section{Handling editor Joseph D Tucker}

Acknowledgements We are grateful to Will Nutland of Prepster and Andrew J Amato-Gauci of ECDC for helpful comments on an earlier draft of this paper; Sheena $\mathrm{McC}$ ormack for providing input on the content of the survey questions; and Lella Cosmaro and Ulrich Marcus for support in translating the survey.

Contributors $T N$ and $A B$ designed the study and survey instrument, with input from AP, GS and AG. The survey was promoted online by AG. Data analysis was performed by $B A$. The manuscript was prepared by $A B$ and $B A$ with input from $T N$, $A P, A G$ and $G S$.

Funding The authors have not declared a specific grant for this research from any funding agency in the public, commercial or not-for-profit sectors.

\section{Competing interests None declared.}

Patient consent Not required.

Ethics approval Ethical approval for this study was obtained from the Research Ethics Committee of La Trobe University (REF: S17-207).

Provenance and peer review Not commissioned; externally peer reviewed.

Open access This is an open access article distributed in accordance with the Creative Commons Attribution Non Commercial (CC BY-NC 4.0) license, which permits others to distribute, remix, adapt, build upon this work non-commercially, and license their derivative works on different terms, provided the original work is properly cited, appropriate credit is given, any changes made indicated, and the use is non-commercial. See: http://creativecommons.org/licenses/by-nc/4.0/.

\section{REFERENCES}

1 European Centre for Disease Prevention and Control/WHO Regional Office for Europe. HIV/AIDS surveillance in Europe 2017 - 2016 data. Stockholm: ECDC, 2017.
2 Phillips AN, Cambiano V, Nakagawa F, et al. Increased HIV incidence in men who have sex with men despite high levels of ART-induced viral suppression: analysis of an extensively documented epidemic. PLoS One 2013;8:e55312.

3 McCormack S, Dunn DT, Desai M, et al. Pre-exposure prophylaxis to prevent the acquisition of HIV-1 infection (PROUD): effectiveness results from the pilot phase of a pragmatic open-label randomised trial. Lancet 2016;387:53-60.

4 Molina JM, Capitant C, Spire B, et al. On-demand preexposure prophylaxis in men at high risk for HIV-1 infection. N Engl J Med 2015;373:2237-46.

5 European Centre for Disease Prevention and Control. Pre-exposure prophylaxis to prevent HIV among MSM in Europe: ECDC Comment. Stockholm: ECDC, 2015.

6 World Health Organisation. Consolidated guidelines on the use of antiretrovial drugs for treating and preventing HIV infection: recommendations for a public health approach. 2nd edn. Geneva: WHO, 2016.

7 European Medicines Agency. First medicine for HIV pre-exposure prophylaxis recommended for approval in the EU: Truvada to enhance existing HIV prevention strategies. London: European Medicines Agency, 2016.

8 European Centre for Disease Prevention and Control. Pre-exposure prophylaxis for HIV prevention in Europe: Evidence brief. Stockholm: ECDC, 2016.

9 PrEP in Europe, 2018. The PrEP situation in Europe London: PrEP in Europe. Available from: http://www.prepineurope.org/en/who-is-the-prep-in-europe-initiative/the-prepsituation-in-europe/

10 Wang X, Nwokolo N, Boffito M. InterPrEP: internet-based pre-exposure prophylaxis (PrEP) with generic tenofovir DF/emtricitabine (TDF/FTC) in London - analysis of pharmacokinetics, safety and outcomes. Glasgow, UK: International Congress on Drug Therapy in HIV Infection (HIV Glasgow), 2016.

11 Melendez-Torres GJ, Bourne A. Illicit drug use and its association with sexual risk behaviour among MSM: more questions than answers? Curr Opin Infect Dis 2016;29:58-63.

12 Gianacas C, Down I, Ellard J. Experiences of HIV: The seroconversion study final report, 2007-2015. Sydney: Kirby Institute, 2015.

13 Collins SP, McMahan VM, Stekler JD. The Impact of HIV Pre-exposure Prophylaxis (PrEP) Use on the Sexual Health of Men Who Have Sex with Men: A Qualitative Study in Seattle, WA. International Journal of Sexual Health 2017;29:55-68.

14 Liu A, Cohen S, Follansbee $S$, et al. Early experiences implementing pre-exposure prophylaxis (PrEP) for HIV prevention in San Francisco. PLoS Med 2014;11:e1001613.

15 Mera Giler R, Magnuson D. Changes in truvada (TVD) for HIV pre-exposure prophylaxis (PrEP) utilization in the United States: (2012-2016). Paris, France: IAS, 2017.

16 AIDES CP. Flash PrEP in Europe online survey: first results. Paris: AIDES \& Coalition Plus, 2017.

17 Kamitani E, Wichser ME, Adegbite AH, et al. Increasing prevalence of self-reported HIV pre-exposure prophylaxis (PrEP) use in published surveys - a systematic review and meta-analysis. AIDS 2018:1.

18 Nic Lochlainn L, O'Donnell K, Hurley C, et al. Using data from a behavioural survey of men who have sex with men (MSM) to estimate the number likely to present for HIV pre-exposure prophylaxis (PrEP) in Ireland, 2017. Eurosurveillance 2017;22:pii=1700768.

19 Association AIDES. What is PrEP [La Prep c'est quoi?]: AIDES, 2016.

20 BHIVA/BASHH. BHIVA/BASHH guidelines on the use of HIV pre-exposure prophylaxis (PrEP). London: British HIV Association and British Association for Sexual Health \& HIV, 2017.

21 Bourne A, Hammond G, Hickson F, et al. What constitutes the best sex life for gay and bisexual men? Implications for HIV prevention. BMC Public Health 2013;13:1083.

22 Smit PJ, Brady M, Carter M, et al. HIV-related stigma within communities of gay men: a literature review. AIDS Care 2012;24:405-12.

23 Golub SA, Gamarel KE, Rendina HJ, et al. From efficacy to effectiveness: facilitators and barriers to PrEP acceptability and motivations for adherence among MSM and transgender women in New York City. AIDS Patient Care STDS 2013;27:248-54.

24 Young I, McDaid L. How acceptable are antiretrovirals for the prevention of sexually transmitted HIV?: A review of research on the acceptability of oral pre-exposure prophylaxis and treatment as prevention. AIDS Behav 2014;18:195-216.

25 Holt M, Murphy DA, Callander D, et al. Willingness to use HIV pre-exposure prophylaxis and the likelihood of decreased condom use are both associated with unprotected anal intercourse and the perceived likelihood of becoming HIV positive among Australian gay and bisexual men. Sex Transm Infect 2012:88:258-63. 